\title{
A Systematic Review of Patient Oncofertility Support Needs in Reproductive Cancer Patients aged 14 to 45 years of age
}

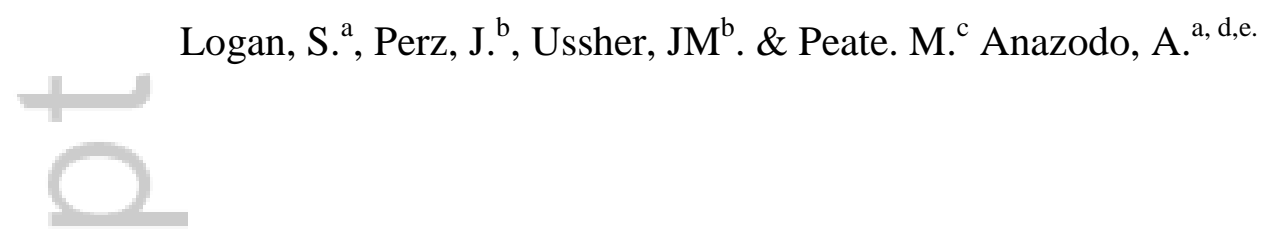

${ }^{a}$ School of Women and Children's Health, Faculty of Medicine, UNSW Australia, Sydney NSW.

${ }^{b}$ Centre for Health Research, School of Medicine, Western Sydney University, Sydney NSW

${ }^{c}$ Psychosocial Health and Wellbeing (emPoWeR) Unit, Department of Obstetrics and

Gynaecology, Royal Women's Hospital, University of Melbourne, Melbourne VIC

'Sydney Children's Hospital, Sydney NSW.

${ }^{\mathrm{e}}$ Prince of Wales Hospital, Sydney NSW

Corresponding author details: Dr Shanna Logan, School of Women and Children's Health, Faculty of Medicine, UNSW Australia, Sydney NSW. Email: shanna.logan@unsw.edu.au

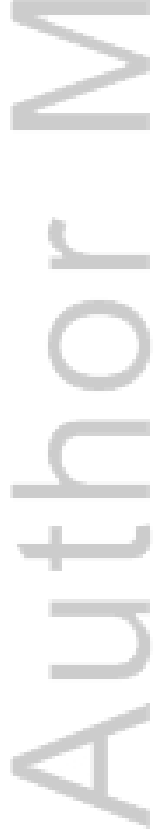

This is the author manuscript accepted for publication and has undergone full peer review but has not been through the copyediting, typesetting, pagination and proofreading process, which may lead to differences between this version and the Version of Record. Please cite this article as doi: $10.1002 /$ pon. 4502

This article is protected by copyright. All rights reserved. 


\begin{abstract}
Objective: Decline in fertility potential brought about by a cancer diagnosis or cancer treatment is one of the biggest impacts to cancer patients' long term quality of life. As such, the current manuscript aimed to systematically review the literature on oncofertility support needs for cancer patients of a reproductive age (14-45 years of age).

Methods: A systematic review of the literature was conducted in May 2016 through the searching of electronic databases Medline, EMBASE, PSYCH Info, Web of Science and SCOPUS, alongside the screening of relevant reference lists. An initial search identified 351 potentially relevant studies. The papers were divided into two categories; papers on patient oncofertility support needs were reviewed for this systematic review and papers on clinician provision of oncofertility support were reviewed for a separate systematic review.
\end{abstract}

Results: A total of 30 studies were included within the final review. Support needs were categorised as information, service, clinician-patient interactions, psychological and family. A number of studies indicated that cancer patients place great important on their oncofertility care and have unmet support needs. Patients were satisfied and felt supported when additional care was taken to ensure fertility information and service needs were met.

Conclusions: Patients desire for clinicians to support their concerns through the provision of adequate information, access to oncofertility services, taking time to discuss oncofertility treatment and concerns, specialised psychological support and responsiveness to individual needs.

Key words: Oncology, Cancer, Support, Fertility, Fertility Preservation, Oncofertility, Patient Needs

Word Count: 5,974

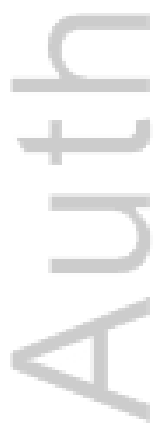

This article is protected by copyright. All rights reserved. 


\section{Background}

National and international guidelines for the treatment of adolescents and young adults with cancer outline the need to discuss infertility risks and fertility preservation options early in patient care. ${ }^{1-4}$ However, research identifies that over half of young adult cancer survivors are unsure of their fertility potential and do not recall their health care provider informing them of the risk for impaired fertility. ${ }^{5}$ Despite fertility preservation playing a valuable role in assisting young men and women to overcome complications of infertility as a result of cancer treatments ${ }^{6}$ fertility preservation rates remain low. ${ }^{7}$ With future pregnancy and parenthood representing normalcy, happiness and life fulfilment for cancer survivors ${ }^{8}$ there is a requirement to assure that oncofertility needs are met throughout the cancer journey.

Recent reviews highlight the benefits of oncofertility interventions in improving quality of life and lowering dissatisfaction in survivorship. ${ }^{9,10}$ Simply undergoing fertility preservation improves the subjective experience of cancer treatments. ${ }^{11}$ The level of general psychological distress in survivorship is related to perceptions of reproductive issues rather than actual fertility status. ${ }^{9}$ Research also suggests that adolescent and young adult cancer patients want to be fully informed about cancer treatment's effects on fertility alongside fertility preservation options; ${ }^{12,13}$ however, historically information needs have not been adequately addressed. ${ }^{14-16}$

Although half of cancer survivors intend to discuss fertility concerns with their clinicians during follow up consultations, significantly fewer engage in these discussions. ${ }^{17}$ In addition, only half of young women who discuss their fertility concerns with their treating doctor report that their concerns were adequately addressed. ${ }^{18}$ Recent research indicates that unmet needs can persist despite general patient satisfaction with information or if fertility preservation procedures were offered. ${ }^{19}$ Therefore, in addition to being offered fertility preservation and fertility information throughout the cancer journey, the way in which these are undertaken may be important in predicting patient satisfaction and feelings of support. It is important to understand the patient experience specifically in receiving oncofertility care, to be able to improve on the provision of services and to ensure that future interventions can be accurately tailored to meet patients' oncofertility support needs. 
Cancer patients of reproductive age (14- 45years of age), which include adolescents and young adults, may not have had a family prior to cancer diagnosis and treatment.

Consequently, this cohort may experience impacted fertility as a greater burden throughout the cancer journey and into survivorship. For example, higher fertility-related concerns and poorer fertility quality of life are seen in cancer survivors of a younger age, and this is related to later distress. ${ }^{20}$ For young adult cancer patients, fertility concerns are related to higher psychological distress, and the experience of cancer influences later reproductive decisions. ${ }^{9}$ It is therefore imperative that cancer patients of reproductive age are supported in their oncofertility care, in order to diminish later impacts to health and quality of life. A review of the current literature would determine the level of oncofertility support that is currently provided to cancer patients of reproductive age. The aim of the current manuscript is therefore to systematically review the literature on oncofertility support needs for cancer patients of reproductive age (14-45 years).

\section{Method}

\section{Inclusion Criteria and Search}

The systematic review considered all studies where oncofertility care and patient support needs were reported from the patient perspective. To meet inclusion criteria cancer patients needed to be within reproductive age (age range reported within 14-45 years or a mean sample age <45 years). Support needs were described as occurring anytime within the cancer journey (pre, during or post cancer diagnosis or treatment). Descriptions of oncofertility interventions or appraisal of oncofertility services or care were also included where there was a patient-reported outcome. Studies needed to be in English, a peer reviewed publication and of sound quality, as assessed through a validated quality assessment tool. ${ }^{21}$ No publication date restrictions were imposed. Any study on the clinician provision of oncofertility support was considered in a separate systematic review to allow us to evaluate the evidence in both areas and enable clearer recommendations. ${ }^{22}$

A literature search was performed in May 2016 through electronic databases Medline, EMBASE, PSYCH Info, Web of Science and SCOPUS, alongside the screening of reference 
lists. Search terms were tailored to individual databases in order to map terms to database subject headings and take an inclusive approach (see Table 1). The search across electronic databases and reference lists identified 351 potentially relevant studies after the deletion of duplicates. All titles and abstracts were screened by a single reviewer. To ensure inter-rater reliability two reviewers assessed the full text of the remaining 85 studies to determine eligibility for inclusion with $100 \%$ consensus reached. A total of 30 studies remained eligible for further analysis (see Figure 1 for inclusion, exclusion flow chart).

\section{Quality Analysis and Extraction}

The quality of final studies was assessed using the Mixed Methods Appraisal Tool (MMAT). ${ }^{21}$ Scores on the MMAT vary from $25 \%$ (one criteria met) to $100 \%$ (all criteria met). Quality was assessed against criteria related to either qualitative or quantitative enquiry. All 30 final studies were of sound quality (75-100\%) and were then analysed for data by two reviewers. Data was extracted from each paper for; characteristics of patients (age of sample, sample size, gender, cancer diagnosis, time since diagnosis); and descriptions and details of support needs.

\section{Results}

\section{Study Characteristics}

Table 2 reflects the key features of each study. Of the total, 17 were quantitative, 12 were qualitative and one utilised mixed methods. Separate studies referring to the same sample of participants were combined on a single row within the data extraction table, unless sample sizes differed, in which case they were reported separately. ${ }^{5,23}$ Where inclusion criteria was met for a sub-group, only the sub-groups data has been analysed. ${ }^{24,25}$ Two studies included paediatric patients within their overall sample $;^{26,27}$ however, the median or mean age was within the 14-45 year age range. The studies were published over an 11 year period, from to 2005-2016 with the median publication year 2012. Three studies, utilising the same sample, were conducted globally. Remaining studies were conducted across a total of nine countries. The sample size range varied from six to 879 participants with large variance attributed to study design. 
The majority of papers (19) reported on female patients' experiences only, followed by a combination of male and female patients (8) or male patients only (3). Across all studies the mean age at time of diagnosis ranged from 27.8 to 39.9 years and at the time of data collection from 23.1 to 46.3 years. Eight papers reported on breast cancer patients only, while other studies reported on a range of cancer diagnoses. One study reporting on the impact of hemotopoietic stem-cell transplantation included a small subsample of non-cancer patients within the analyses $(n=3,1.5 \%$ of the overall sample). Three studies did not report the specific cancer diagnoses of patients, reporting that the diagnosis varied ${ }^{28}$ or failed to report any information. ${ }^{29,30}$ Eight studies did not report the time between diagnosis and data collection. Of those that did report this information, data collection ranged from prior to treatment commencing or one month post-diagnosis, to 15 years post diagnosis. However, the majority of studies reported on patients who were $<5$ years post treatment, with only seven studies including time since diagnosis at $>5$ years post diagnosis. ${ }^{26,31-34}$ Five studies reported on a fertility patient intervention ${ }^{29,32,33,35,36}$ and the impact this had on patient's experiences with oncofertility care or fertility knowledge. Support needs were extracted from studies under the following categories: information, fertility service, clinician-patient interactions, psychological support, or family and social supports; with category definitions guided by the data extracted. ${ }^{31,37}$

\section{Support Needs}

\section{Information.}

Receiving information about fertility was regarded as important, as reported in four studies. ${ }^{38-}$ ${ }^{41}$ The majority of adolescent and young adult male and female patients (17-35 years) who are less than five years post-diagnosis ranked receiving more information as 'very important' ${ }^{38}$ Similarly, recently diagnosed female breast cancer patients regarded fertility information to be 'extremely important' $(65 \%)$ or 'very important' $(22 \%) .{ }^{40}$ Women who were younger, had plans for child bearing at diagnosis, had no children at diagnosis or single were more likely to rate fertility information as highly important. ${ }^{39,41}$ 
Dissatisfaction with the inadequate provision of fertility information was reported in seven studies published 2007-2015. 24,25,27,30,32,39,42 A large mixed gender sample of adolescents and young adults (14-24 years) treated at a speciality cancer centre reported that the provision of fertility information was poor and not age appropriate. ${ }^{27}$ A study of adult female breast cancer patients also reported fertility and menopause literature did not cater to younger women in both its language use and images. ${ }^{24}$ Less than half (41-44\%) of adult female breast cancer patients received information about fertility from their treating medical professional, and of those approximately $16-19 \%$ were dissatisfied. ${ }^{42}$ In another study, three young women (12-24 years) reported receiving inadequate fertility information, that information was withheld, or that they were not informed by their clinician at all, and this was an issue they felt needed addressing. ${ }^{30}$ Female patients with treatment induced infertility reported that they were not informed of infertility risk before treatment and were dissatisfied with medical information provided during treatment by their treating physician; with lack of information associated with older age and non-hormone based treatment. ${ }^{25}$ In a sample of women aged 10-50 years at diagnosis, being younger, childless, wanting a child and having a lower quality of life predicted dissatisfaction with fertility information. ${ }^{39}$ Additionally, fertility knowledge deficits were reported to persist regardless of cancer treatment status (not started, receiving, or completed), ${ }^{32}$ indicating that women continued to feel uninformed into survivorship.

Three studies indicated that newly diagnosed adult female patients seek out additional fertility information. ${ }^{36,37,40}$ The majority of female patients held preference for as much information as possible (74\%), wished to know both the good and bad news (73\%), and were 'moderately-very active' in seeking out information. ${ }^{40}$ Most women searched the internet and sought information on other women's experiences regarding a range of fertility-related topics, however reported concerns that information on the internet was unreliable. ${ }^{37}$ Although $61 \%$ accessed the internet for additional fertility information only $2 \%$ found all information needed. ${ }^{36}$ Women also reported a strong desire for fertility medical information on topics such as treatment risks, benefits, success rates and costs. ${ }^{37}$

Patient unmet need for fertility information and support was identified in 10 studies published 2008-2013. 5,23,24,26,30,32,35,37,43,44 Adolescent cancer patients (13-20 years) reported very little 
prior fertility knowledge ${ }^{26}$ and do not know where they could obtain this information in the future (12-24 years). ${ }^{30}$ Adult women 1-3 years post diagnosis reported having only 'a little' information on infertility treatment and resources, ${ }^{32,36}$ with only $11 \%$ believing they received sufficient information. ${ }^{36}$ Patients desired written information that reflected decent literature,${ }^{37,43,44}$ was straight forward and non-conflicting, ${ }^{24}$ and given at the time of diagnosis. ${ }^{37}$ The majority (65-68\%) of male and female patients desired information about infertility and options for having children, with unmet information needs reflected in $42 \%$ of the sample. ${ }^{5,23}$ Younger age (18-29 years), being unmarried, non-white, female ${ }^{5}$ or having reoccurring cancer illness, ${ }^{23}$ was associated with having more unmet information needs. Four studies reported on the timing of information provision. ${ }^{26,31,41,43}$ Adolescent and young adult patients (13-20 years) strongly supported being informed with broad fertility information at the time of diagnosis, and being supplied with greater detail at a later point in time. ${ }^{26}$ Adolescent and adult men (15-41 years) felt overwhelmed by the amount of cancer information at the time of diagnosis and were unclear about the implication of their treatment on future fertility. ${ }^{31}$ Adult female patients also endorsed the need for information shortly after news of diagnosis, to allow time to absorb information. ${ }^{43} \mathrm{~A}$ second study with women highlighted the importance of receiving fertility information during cancer treatment decision-making and in follow-up, rather than at diagnosis, during, or end of treatment. ${ }^{41}$

Ten recent studies (published 2012-2016) reported on satisfaction with information provision. $^{29,30,33,35,36,40,45-48}$ Adolescent and young adult male patients (12-24 years) felt information was thorough, comprehensive and useful. ${ }^{30}$ Young adult female patients (18-26 years at study) reported that the provision of information prior to treatment was really helpful, that they felt satisfied and reassured at being an informed patient. ${ }^{48}$ Recently diagnosed adult female breast cancer patients who received written materials rated it 'useful to very useful' ${ }^{47}$ and higher fertility knowledge was associated with lower levels of decisional conflict (uncertainty). ${ }^{40}$ Information materials were deemed crucial by young women making decisions on cancer treatment type (18-26 years $)^{48}$ and fertility preservation (mean age 28.3 years). ${ }^{45}$ One study found that $53 \%$ of female adult patients provided with 
information about fertility were satisfied; with greater satisfaction at four years postdiagnosis associated with having had the opportunity to ask all desired questions at diagnosis and being satisfied with their medical follow-up. ${ }^{46}$ One intervention study reported that $70 \%$ of adult female patients were completely satisfied with the exhaustiveness and clarity of information received, ${ }^{35}$ while another intervention was found to have a significant increase in fertility knowledge six months post intervention. ${ }^{33,36} \mathrm{~A}$ third intervention had no impact on sperm banking knowledge but did significantly reduce men's decisional conflict. ${ }^{29}$

\section{Fertility Service.}

Eight studies reported the importance of being given the opportunity to pursue fertility preservation, or need for fertility services. ${ }^{5,23,26,35,38,40,43,49}$ Young cancer patients (11-20 years) perceived the offer of fertility preservation as a professional belief of a positive prognosis. ${ }^{26}$ Interestingly, young adult female patients (17-35 years) rated fertility preservation methods as more important than young males. ${ }^{38}$ Generally, fertility treatments were viewed positively by newly diagnosed adult female cancer patients. ${ }^{40}$ Fertility preservation was felt to be an important part of cancer therapy (54.2\%), an 'open window toward the future' $(58.3 \%)$ and an 'option not to be wasted' $(70.8 \%) .{ }^{35}$ One in five adult women reported fertility preservation discussions were important at the time of diagnosis and treatment planning, while $70 \%$ of women had continued interest in discussing fertility preservation post treatment. ${ }^{49}$ Female adult patients reported the need to be able to access a fertility expert with specialised information and cancer training, for clinicians to acknowledge the importance of fertility, and additional appointments to those who need to discuss fertility at another time. ${ }^{43}$ A large mixed gender sample showed that $38-40 \%$ indicated the need for fertility treatment, with unmet service needs reported by $62 \% .^{5,23}$ Unmet infertility treatment/service needs were associated with being unmarried, younger age (18-29 years) or having daily health interference. ${ }^{5}$

Four studies reported on fertility preservation and service use for adult female patients published 2012-2016..$^{37,47,49,50}$ The proportions of women referred or encouraged to speak with a fertility specialist or service ranged from $14 \%-67 \% .{ }^{41,47,49}$ The preferred method for 
receiving education was individual consultation. ${ }^{41}$ In the case where $67 \%$ of newly diagnosed female breast cancer patients were referred for fertility preservation, 59\% attended a fertility consultation and women over 35 years were significantly more likely to attend. ${ }^{47}$ Barriers for patients include lack of access to fertility treatment centres dues to geographical area, and limited access to fertility preservation technologies (e.g. only embryo freezing available).$^{51}$

Two studies reported on male fertility preservation sperm banking practices, published 2007 and 2011. ${ }^{28,31}$ Male patients regarded access to sperm banking as important, ${ }^{28,31}$ however one study reported the facilities were 'soulless'. ${ }^{28}$ Banking sperm provided a safety net and assurance of normal life post treatment for male patients (15-41 years), with appointments kept like other clinical appointments for cancer treatment. ${ }^{31}$ However, these men reported little understanding of treatment impacts to fertility, how sperm may be used in the future, or obligations to make decisions about ongoing sperm storage, management or disposal. Without the practical assistance of their treating clinicians in making fertility preservation appointments these men would not have banked sperm. Moreover, given the lack of understanding fertility monitoring was perceived as an intrusion into everyday life.

Five studies reported on the use of fertility preservation counselling (2007-2015). ${ }^{27,28,30,34,45}$ Young women $(\leq 29$ years) who wished to conceive in the future placed importance on fertility preservation counselling. ${ }^{45}$ For female patients in survivorship, uncertain prognosis (29.2\%), risk of cancer recurrence $(20.7 \%)$, parity $(16.4 \%)$, age $(12.1 \%)$ or unknown desire for future fertility (4.9\%) may have limited the amount of fertility counselling that was received. ${ }^{34}$ Three studies reported mixed results utilising adolescent and young adult samples. In one study all young men were offered fertility preservation and referred for fertility preservation counselling; however, all young women were not. ${ }^{30}$ In a second study of mixed gender $34 \%$ received fertility preservation counselling; however, $36 \%$ were not told of the risks to fertility before treatment commenced. ${ }^{27}$ While in a third study, counselling was not offered to males, despite a strong desire for it to be available. ${ }^{28}$

\section{Clinician-Patient Interactions.}


Four studies reported on rates of clinician-patient fertility discussions with adult female patients (2005-2016)..$^{39,41,47,49}$ In a 2005 study, $71 \%$ of breast cancer patients reported discussing fertility with a clinician, with $45 \%$ of these patients initiating the discussion themselves. ${ }^{41}$ However, in a more recent 2016 study almost all (93\%) recently diagnosed breast cancer patients recalled their oncology clinician initiating a fertility discussion prior to treatment, with no association between the clarity of spoken information and likelihood of attending a fertility consultation. ${ }^{47}$ The majority (68\%) of recently diagnosed patients in a third 2012 study also reported discussing fertility with a clinician, with approximately one third of the overall sample indicating a personal interest in discussing fertility and placing importance on fertility discussions. ${ }^{49}$ In addition, $38 \%$ of female patient survivors recalled discussing fertility with their clinician since diagnosis. ${ }^{39}$

Four studies reported on the importance of the clinician-patient interaction in decision making. ${ }^{37,40,45,50}$ The majority of adult female breast cancer patients held a preference for making decisions after seriously considering the clinician's opinion (40\%), or having shared responsibility for decision making with the clinician $(35 \%) .{ }^{40}$ Decisional support from medical staff was regarded as important in making decisions about fertility preservation counselling for adult female patients regardless of whether they proceeded with fertility preservation. ${ }^{45}$ When decisional support by medical staff was done well it reflected active listening; open, honest, non-judgemental communication; in an environment that promoted respect; empowered women to discuss personal thoughts and emotions; trustworthiness; alongside efforts to decrease stress or tension. ${ }^{37,50}$ Factors that impeded decisional debriefing included being insensitive, judgemental and holding a perceived hidden agenda. ${ }^{37}$

Three studies reported on positive and supportive clinician-patient fertility interactions. ${ }^{26,35,37}$ Young cancer patients (13-20 years) in survivorship recalled satisfaction with how they were informed of fertility risk when the discussion reflected professional sensitivity, friendliness, lack of embarrassment and clarity of information. ${ }^{26}$ These patients valued a clinicians' professional willingness to offer advice and encouragement, and that the final decision regarding fertility treatment rested with the patient. Clinicians who were able to incorporate effective and efficient communication systems between multiple clinicians enhanced 
women's ability to obtain and integrate information. ${ }^{37}$ One study reported on the outcome of a fertility preservation intervention. The majority of patients enrolled on the program rated both their doctors' empathy and the collaboration between the fertility and oncology clinicians as 'absolutely satisfying'. ${ }^{35}$

However, six studies reported on poor clinician-patient fertility interactions and dissatisfaction with support provided (2007-2014). ${ }^{24,28,30,34,37,43}$ Young men (16-26 years) reported the desire to discuss fertility in greater details and when a doctor was uncomfortable discussing fertility this was awkward for one patient. ${ }^{28}$ Young female patients (12-24 years) reported a range of negative individual experiences. ${ }^{30}$ One woman felt rushed by her clinician, desired respect and reported information was conveyed in an insensitive manner, while a second was told preservation was not an option with no further explanation, resulting in later distress. Adult female patients expressed a desire to speak with a female clinician, reporting frustration and a perception that male clinicians could not understand female symptoms. ${ }^{24}$ Another study of recently diagnosed female patients reported that fertility preservation was not addressed or information was inadequate, that clinicians were lax in addressing fertility concerns, clinicians were unable or unwilling to provide advice specific to a patient's situation, and an increased difficulty when two clinicians gave opposing views. ${ }^{37}$ Female cancer survivors also perceived that they were told discrepant information from different clinicians and that their reproductive choices were denied by clinicians making fertility decisions on their behalf. ${ }^{43}$ These patients also desired to be informed about all fertility factors by clinicians so that they could make an informed choice to be treated as intelligent. Female cancer survivors also recalled that clinicians focused on the negative aspects of fertility preservation. ${ }^{34}$ In this sample, communication barriers included omission of information, nondisclosure of risks to reproductive health, or providing incorrect information on fertility preservation options.

\section{Psychological Support}

Seven studies described the importance and psychological impact of considering impacted fertility. ${ }^{24,25,35,38,39,50,51}$ Fertility and family planning were regarded as a significant issue for 
women, ${ }^{24}$ with fertility preservation regarded as the most difficult decision women had ever made. ${ }^{50}$ Infertility was a major concern for both men and women, with more than $70 \%$ reporting fertility issues. ${ }^{51}$ Young adult female patients (17-35 years) rated risk of infertility as more important than males ${ }^{38}$ and women with higher anxiety rated fertility information as more important. ${ }^{39}$ As such, female patients expressed the desire for additional support in way of a specialised psychological service ${ }^{25}$ or a post treatment internet group. ${ }^{24}$ An intervention study found that $71 \%$ of patients who participated in a fertility preservation program considered the presence of a psychologist helpful and rated the collaboration between a psychologist and gynaecologist as 'absolutely satisfying' (70.9\%). ${ }^{35}$

Adolescent and young adult men and women (13-20 years) reported strong adverse reactions when clinicians, who initiated the fertility discussion, assumed that fertility would be of little consequence. ${ }^{26}$ Similarly, recently diagnosed women reported discomfort, sadness, embarrassment and frustration at clinicians' dismissal of patients' desire for information; dissatisfaction when fertility was not addressed directly; anger and annoyance when the clinician was impersonal; and feeling uncomfortable, sad and embarrassed when clinicians dismissed the importance of fertility. ${ }^{48}$ It was also a common experience for women to feel overwhelmed $^{50}$ and to feel anger and sadness at the lack of control over dissemination of personal fertility information. ${ }^{48}$ Young men (16-26 years) reported feeling annoyed and devastated at not having the opportunity to bank sperm or be informed. ${ }^{28}$

Coming to terms with the impact of cancer on fertility was described in three studies. Young patients (13-20 years) found it difficult to separate the lack of current desire for parenthood from potential to do so in the future. ${ }^{26}$ Overall, women reported that cancer robs them of the natural choice to have a biological child, while women who had children already at the time of diagnosis felt their fertility concerns were neglected by clinicians. ${ }^{43}$ Fears held by female patients included the health of frozen oocytes/ovarian tissue (37.5\%), ability for a future pregnancy $(37.5 \%)$, not surviving to use stored samples $(12.5 \%)$ and fertility preservation increasing the risk of cancer $(4.2 \%){ }^{35}$ 
Studies also described the psychological impact of impaired fertility after treatment. Treatment-induced infertility was associated with lower physical and mental quality of life, alongside increased negative consequences of treatment on sexual life. ${ }^{25}$ Loss of fertility posed uncertainty and often tremendous heartache and grief for female cancer patients. ${ }^{37}$ Feelings of isolation were reported by women suffering early menopause relative to age matched peers. ${ }^{24}$ However, information about recovered fertility contributed toward feelings of restored masculinity for men in survivorship. There were perceived positive psychological benefits to having banked sperm - it was seen as a meaningful experience - and fears of disease reoccurrence influenced decisions to not dispose of sperm post-treatment. ${ }^{31}$ For male patients, sperm monitoring was attended with the hope of fertility reassurance, while nonattendance of monitoring was a result of anxiety surrounding fertility potential. ${ }^{31}$

\section{Family and Social Support.}

Generally, support from family and others was important for making decisions about fertility preservation counselling for women with breast cancer. ${ }^{45}$ Patients often engaged with clinicians, parents and friends in fertility preservation decisional debriefing ${ }^{50}$ and believed that the consultation with a religious leader could aid in determining preferences, value formation and clarification. ${ }^{37}$ However, engaging with committed partners sometimes resulted in difficulties obtaining agreement about cryopreservation..$^{50}$

Support from one or two medical professionals alongside family support was the preference in an adolescent and young adult (13-20 years) mixed gender sample. ${ }^{26}$ Although one young male reported he was satisfied with his parents withholding fertility information until after treatment, to save him additional distress, ${ }^{30}$ overall there was strong support for professionals to talk directly with adolescent and young adult patients (12-24 years) rather than to or through parents. ${ }^{26,30}$ Disagreement between parents and patients around preservation preference resulted in distress for all parties, ${ }^{26}$ and lead to pressure to conform to parental desire or occasionally for patients to opt for a parent to not be present during consultation to not influence the patient's decision. ${ }^{37}$ Male patients (15-41 years) often reported embarrassment when forced to bank sperm, or discuss fertility with parents; however, they 
regarded the presence of a parent or partner helpful in fertility monitoring consultations post cancer treatment. $^{31}$

\section{Discussion}

The aim of the current manuscript was to systematically review the literature on oncofertility support needs for cancer patients of reproductive age. Given the potential for lowered quality of life and heightened psychological distress in cancer patients of reproductive age ${ }^{20}$ when fertility needs are not considered, it is important to determine whether patients are currently supported in the oncofertility care that they desire. Support needs were categorised into information, fertility service, clinician-patient interactions from a patient perspective, psychological support, or family and social support.

Although some recent literature does indicate that fertility information provision may be improving, a number of studies indicate that currently patients need more support in their fertility information needs. Almost half of male and female patients of reproductive age report unmet infertility information needs, with younger age or female patient status associated with greater unmet needs. ${ }^{5,23}$ Inadequate information provision, age-inappropriate written materials or dissatisfaction with information provided were common difficulties, ${ }^{37,39}$ resulting in patients having to seek out additional information in an attempt to have their needs met. ${ }^{37,40}$ This analysis indicates that patients hold a preference for being informed of fertility in greatest detail post diagnosis and prior to treatment commencing; $;^{31,41,43}$ with this information integral in treatment decision making for both cancer treatment ${ }^{48}$ and fertility preservation. ${ }^{45}$ Given the success of fertility information interventions on patient satisfaction ${ }^{35}$ or level of fertility knowledge, ${ }^{33,36}$ these deficits may be corrected if clinicians are able to implement a change in information provision practices.

This review highlights the importance of the availability of oncofertility services, with fertility preservation regarded as both a welcome expression of a life after cancer ${ }^{26}$ and an important part of a patients' cancer care. ${ }^{35}$ Guidelines reflect impairment of fertility due to oncological treatment should be mentioned at time of diagnosis and patients should be referred to a specialist as soon as possible. ${ }^{1,2}$ However, the proportion of women who are referred for fertility 
preservation varies greatly from $14-67 \%,{ }^{47,49}$ indicating a lack of consistency in implementing this best practice procedure. Although men appear to express lower support needs in accessing fertility preservation services, ${ }^{30}$ male patients describe having limited understanding of sperm management practices or ongoing fertility monitoring; ${ }^{31}$ indicating a need for clinicians to better describe the implications of fertility preservation to this cohort.

There was also a greater need for the service of fertility preservation counselling, identified by both male and female patients, with a number of factors shown to limit this; such as younger age, current parenthood or unknown desire for a future pregnancy. ${ }^{34}$ Given fertility preservation can take up to three weeks in female patients, a delay in fertility preservation counselling may incur a delay in oncological treatment. As such, fertility preservation counselling should be performed as soon as possible after cancer diagnosis.

Patient support needs are often unmet during clinician-patient interactions, with clinicians not fully considering patient's fertility concerns or not able to provide a personalised approach. ${ }^{24,30,34,37}$ Given patients place great importance on discussing fertility with their clinician in order to aid in treatment decision making ${ }^{40,45}$ it is imperative that clinicians are able to meet patients' needs within clinician-patient interactions. Limited research does report on positive interactions where clinicians are supportive, provide clear communication, ${ }^{26,37}$ and additional time is taken to discuss fertility concerns. However, the majority of research indicates that patients are dissatisfied with the fertility discussions that they have with their treating clinicians. Considering the importance of timing around fertility preservation and cancer treatment, there is an onus on clinicians to initiate fertility discussions and better identify if patients wish to discuss fertility in greater detail, and to provide additional time to engage in such consultations.

This review indicates that female patients perceive more barriers in accessing fertility preservation services and experience fewer positive fertility discussions with their clinicians. ${ }^{30}$ It is likely that this lower referral rate is due in part to the different clinical procedures required for fertility preservation in females; which can result in a delay to start cancer treatment, ${ }^{52-54}$ are more invasive, and pose greater ethical concerns and contra- 
indications, ${ }^{55}$ compared to some male preservation procedures. However, some research does indicate that clinicians feel more comfortable in discussing fertility preservation with male patients $^{56}$ and appropriate access to facilities may hinder female patient referral. ${ }^{53,57}$ Yet given that women are more likely to place importance on infertility compared to males, ${ }^{38}$ female patients need to be adequately supported in addressing their fertility needs.

Cancer patients of reproductive age experience strong emotional reactions when being informed of the risk for future infertility or when fertility concerns are not addressed adequately. ${ }^{26,28,37,48}$ One intervention study showed that the collaboration between a psychologist and other clinicians was a helpful approach, ${ }^{35}$ perhaps not surprising given the significant psychological impact that actual or threatened impaired fertility has for many patients. ${ }^{24,26,35,37,43}$ There is therefore a need to ensure that patients are supported emotionally by clinicians when providing oncofertility care. Psychological counselling is a required support $^{25}$ that may assist in the provision of comprehensive care. A review of cancer patients' fertility-related psychological distress is warranted, to better determine the scope and nature of this support need and tailor care accordingly.

Lastly, results indicate that the involvement of family or additional persons can be useful in providing patient support, assisting in fertility decision making, or when attending fertility follow up consultations. ${ }^{31,37,45,50}$ However, for young patients there is a strong desire that clinicians address fertility with them directly and not through parents, ${ }^{26,30}$ whose occasional opposing views on oncofertility care can add additional stress. ${ }^{31,37}$ It is imperative that clinicians support young patients of reproductive age in discussing and making decisions regarding their oncofertility treatment, and for clinicians to be guided by patients in the way to include family members to provide additional support. Although not captured within this review, it is also likely that impaired fertility will impact on partner relationships, with patients fearing negative relational consequences. ${ }^{58,59}$ As such, additional support may need to be provided within a relational framework to both patients and their partners.

Study Limitations

This article is protected by copyright. All rights reserved. 
As the majority of literature to date either reports on newly diagnosed patients, or those in long-term survivorship (up to 15 years following treatment), this review is limited in its ability to accurately determine how the documented support needs compare to those in active treatment, or in recent survivorship. Regardless, this review has identified that the majority of patients place importance on the provision of oncofertility information, access to services, alongside clear and open communication with their clinicians regarding their future fertility. Given that fertility is equally important at the time of treatment planning and follow up ${ }^{41}$ and that psychological distress related to fertility presents at time of diagnosis ${ }^{48}$ and persists into survivorship, ${ }^{31}$ it is important to ensure that support is offered to all patients of reproductive age throughout the entire cancer journey.

\section{Clinical Implications}

Based on the findings of this review it is important for future research and clinical practice to consider the level of information that is supplied to patients, to consider the timing of when this information is delivered in relation to treatment planning and to be responsive to individual patients' fertility concerns. Taking additional time to consult with patients about their fertility and options for care would ensure that patients are supported in making treatment decisions; alongside the provision of age appropriate written material that patients may keep with them to refer to throughout the treatment planning process.

Access to fertility preservation services, a fertility specialist, fertility counselling or specialised psychological service at all points of the cancer journey would aid in increasing the support that patients' receive and serve to lower the emotional burden of impaired fertility. It is equally important that treating oncology clinicians fully consider patients' fertility needs within their patient encounters. Clinicians should aim to be qualified and open to discuss patient fertility concerns and be individually responsive to patient's fertility requirements, in information provision, access to services and involvement with family.

The results of this review will be utilised in the development of an international competency framework which will assist in providing a global guideline for treating clinicians in the provision of oncofertility care to cancer patients. As such, these results have the potential to 
translate to clinical practice; by informing on the way in which services are currently provided to cancer patients of reproductive age and provide the opportunity for improvements in oncofertility support and care, via the provision of updated treatment guidelines.

Funding Source: This work is supported by Kids Cancer Alliance (KCA), a CINSW Translational Cancer Research Centre (15/TRC/1-04).

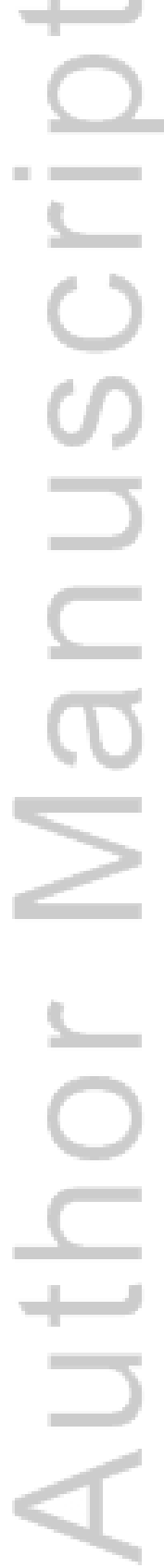

This article is protected by copyright. All rights reserved. 


\section{References}

1. Fertility: Assessment and treatment for people with fertility problems (NICE clinical guideline CG156). London2013.

2. Fertility preservation for AYAs diagnosed with cancer: Guidance for health professionals. Sydney: Cancer Council Australia;2014.

3. Loren AW, Mangu PB, Beck LN, et al. Fertility preservation for patients with cancer: American Society of Clinical Oncology clinical practice guideline update. J Clin Oncol. Jul 1 2013;31(19):2500-2510.

4. NCNN Clinical Practice Guidelines in Oncology for Adolescent and Young Adult (AYA) Oncology (Version I.2012). Fort Washington, PA2011.

5. Zebrack B. Information and service needs for young adult cancer survivors. Support Care Cancer. April 2009;17(4):349-357.

6. Klonoff-Cohen H. Establishing a fertility preservation database: No time like the present. Expert Review of Obstetrics and Gynecology. 2012;7(3):213-225.

7. Petroganagos A, Campo-Engelstein L. Tough Talk: Discussing fertility preservation with adolescents and young adults with cancer. Journal of Adolescent and Young Adult Oncology. 2015;4(3):96-99.

8. Gonçalves V, Sehovic I, Quinn G. Childbearing attitudes and decisions of young breast cancer survivors: a systematic review. Hum. Reprod. Update. March 1, 2014 2014;20(2):279-292.

9. Sobota A, Ozakinci G. Fertility and parenthood issues in young female cancer patients-a systematic review. J. Dec 2014;8(4):707-721.

10. Deshpande K, Braun IM, Meyer FL. Impact of Fertility Preservation Counselling and Treatment on Psychological Outcomes Among Women with Cancer: A Systematic Review. Cancer. 2015;15:3939-3947.

11. Treves R, Grynberg M, le Parco S, Finet A, Poulain M, Fanchin R. Female fertility preservation in cancer patients: an instrumental tool for the envisioning a postdisease life. Future Oncol. May 2014;10(6):969-974.

12. Barlevy D, Wangmo T, Elger BS, Ravitsky V. Attitudes, Beliefs, and Trends Regarding Adolescent Oncofertility Discussions: A systematic literature review. Journal of Adolescent and Young Adult Oncology. 2016;5(2):119-134.

13. Pacey A, Merrick $\mathrm{H}$, Arden-Close $\mathrm{E}$, et al. Implications of sperm banking for health-related quality of life up to 1 year after cancer diagnosis. Br J Cancer. Mar 19 2013;108(5):10041011.

14. Peate M, Meiser B, Hickey M, Friedlander M. The fertility-related concerns, needs and preferences of younger women with breast cancer: a systematic review. Breast Cancer Res. Treat. Jul 2009;116(2):215-223.

15. Grover NS, Deal AM, Wood WA, Mersereau JE. Young Men With Cancer Experience Low Referral Rates for Fertility Counseling and Sperm Banking. J Oncol Pract. May 2016;12(5):465-471.

16. Quinn GP, Murphy D, Knapp C, et al. Who decides? Decision making and fertility preservation in teens with cancer: a review of the literature. The Journal of adolescent health : official publication of the Society for Adolescent Medicine. Oct 2011;49(4):337-346.

17. Absolom K, Eiser C, Michel G, et al. Follow-up care for cancer survivors: views of the younger adult. Br J Cancer. Aug 2009;101(4):561-567. 
18. Partridge $\mathrm{AH}$, Gelber S, Peppercorn J, et al. Web-based survey of fertility issues in young women with breast cancer. J Clin Oncol. Oct 2004;22(20):4174-4183.

19. Garvelink MM, ter Kuile MM, Bakker RM, et al. Women's esperiences with information provision and deciding about fertilty preservation in the Netherlands: 'satisfaction in general, but unmet needs'. Health Expectations. 2013;18:956-968.

20. Howard-Anderson J, Ganz PA, Bower JE, Stanton AL. Quality of life, fertility concerns, and behavioral health outcomes in younger breast cancer survivors: a systematic review. J Nat/ Cancer Inst. Mar 7 2012;104(5):386-405.

21. Pluye P, Gagons M-P, Griffiths F, Johnson-Lafleur J. A Scoring System for Appraising Mixed Methods Research , and Concomitantly Appraising Qualitative, Quantitative and Mixed Methods Primary Studies in Mixed Studies Reviews. International Journal of Nursing Studies. 2009;46(4):529-546.

22. Logan S, Perz J, Ussher JM, Peate M, Anazodo A. Clinician Provisions of Fertility Support in Paediatric and Young Adult Cancer Patients of a Reproductive Age: A systematic review. Psychooncology. Under Review.

23. Zebrack B. Information and service needs for young adult cancer patients. Support Care Cancer. Dec 2008;16(12):1353-1360.

24. Anderson DJ, Yates P, McCarthy A, et al. Younger and older women's concerns about menopause after breast cancer. Eur J Cancer Care (Engl). Nov 2011;20(6):785-794.

25. Mancini J, Rey D, Préau M, Malavolti L, Moatti J-P. Infertility induced by cancer treatment: inappropriate or no information provided to majority of French survivors of cancer. Fertility and Sterility. 11// 2008;90(5):1616-1625.

26. Crawshaw MA, Glaser AW, Hale JP, Sloper P. Male and female experiences of having fertility matters raised alongside a cancer diagnosis during the teenage and young adult years. Eur $J$ Cancer Care (Engl). Jul 2009;18(4):381-390.

27. Smith S, Davies S, Wright D, Chapman C, Whiteson M. The experiences of teenagers and young adults with cancer - Results of 2004 conference survey. Eur J Oncol Nurs. Sep 2007;11(4):362-368.

28. Chapple A, Salinas M, Ziebland S, McPherson A, Macfarlane A. Fertility Issues: The Perceptions and Experiences of Young Men Recently Diagnosed and Treated for Cancer. Journal of Adolescent Health. Jan 2007;40(1):69-75.

29. Huyghe E, Martinetti P, Sui D, Schover LR. Banking on Fatherhood: pilot studies of a computerized educational tool on sperm banking before cancer treatment. Psychooncology. Sep 2009;18(9):1011-1014.

30. Wright $\mathrm{Cl}$, Coad J, Morgan S, Stark D, Cable M. 'Just in case': the fertility information needs of teenagers and young adults with cancer. Eur J Cancer Care (Engl). Mar 2014;23(2):189198.

31. Eiser C, Arden-Close E, Morris K, Pacey AA. The legacy of sperm banking: how fertility monitoring and disposal of sperm are linked with views of cancer treatment. Hum Reprod. Oct 2011;26(10):2791-2798.

32. Jukkala AM, Azuero A, McNees P, Bates GW, Meneses K. Self-assessed knowledge of treatment and fertility preservation in young women with breast cancer. Fertil Steril. Nov 2010;94(6):2396-2398.

33. Meneses K, McNees P, Azuero A, Jukkala A. Evaluation of the Fertility and Cancer Project (FCP) among young breast cancer survivors. Psychooncology. Oct 2010;19(10):1112-1115. 
34. Niemasik EE, Letourneau J, Dohan D, et al. Patient perceptions of reproductive health counseling at the time of cancer diagnosis: a qualitative study of female California cancer survivors. J. Sep 2012;6(3):324-332.

35. Razzano A, Revelli A, Delle Piane L, et al. Fertility preservation program before ovarotoxic oncostatic treatments: role of the psychological support in managing emotional aspects. Gynecol Endocrinol. Nov 2014;30(11):822-824.

36. Meneses K, McNees P, Azuero A, Jukkala A. Development of the Fertility and Cancer Project: an Internet approach to help young cancer survivors. Oncol Nurs Forum. Mar 2010;37(2):191-197.

37. Hershberger PE, Finnegan L, Altfeld S, Lake S, Hirshfeld-Cytron J. Toward theoretical understanding of the fertility preservation decision-making process: examining information processing among young women with cancer. Research and theory for nursing practice. 2013;27(4):257-275.

38. Gupta AA, Edelstein K, Albert-Green A, D'Agostino N. Assessing information and service needs of young adults with cancer at a single institution: the importance of information on cancer diagnosis, fertility preservation, diet, and exercise. Support Care Cancer. Sep 2013;21(9):2477-2484.

39. Nakayama K, Liu P, Detry M, et al. Receiving information on fertility- and menopause-related treatment effects among women who undergo hematopoietic stem cell transplantation: changes in perceived importance over time. Biol Blood Marrow Transplant. Nov 2009;15(11):1465-1474.

40. Peate $M$, Meiser $B$, Friedlander $M$, et al. It's now or never: fertility-related knowledge, decision-making preferences, and treatment intentions in young women with breast cancer-an Australian fertility decision aid collaborative group study. J Clin Oncol. May 1 2011;29(13):1670-1677.

41. Thewes B, Meiser B, Taylor A, et al. Fertility- and menopause-related information needs of younger women with a diagnosis of early breast cancer. J Clin Oncol. Aug 2005;23(22):51555165.

42. Miyashita M, Ohno S, Kataoka A, et al. Unmet Information Needs and Quality of Life in Young Breast Cancer Survivors in Japan. Cancer Nurs. Nov-Dec 2015;38(6):E1-E11.

43. Lee RJ, Wakefield A, Foy S, Howell SJ, Wardley AM, Armstrong AC. Facilitating reproductive choices: The impact of health services on the experiences of young women with breast cancer. Psychooncology. Oct 2011;20(10):1044-1052.

44. Olsson $M$, Jarfelt $M$, Pergert $P$, Enskar K. Experiences of teenagers and young adults treated for cancer in Sweden. Eur J Oncol Nurs. Oct 2015;19(5):575-581.

45. Baysal O, Bastings $\mathrm{L}$, Beerendonk CCM, et al. Decision-making in female fertility preservation is balancing the expected burden of fertility preservation treatment and the wish to conceive. Hum Reprod. 2015;30(7):1625-1634.

46. Ben Charif A, Bouhnik AD, Rey D, et al. Satisfaction with fertility- and sexuality-related information in young women with breast cancer-ELIPPSE40 cohort. BMC Cancer. August 05 2015;15 (1) (no pagination)(572).

47. Cohen L, Hamer J, Helwig C, et al. Formal evaluation of PYNK: Breast Cancer Program for Young Women-the patient perspective. Current Oncology. Apr 2016;23(2):E102-E108.

48. Dryden A, Ussher JM, Perz J. Young women's construction of their post-cancer fertility. Psychol Health. 2014;29(11):1341-1360. 
49. Scanlon M, Blaes A, Geller M, Majhail NS, Lindgren B, Haddad T. Patient Satisfaction with Physician Discussions of Treatment Impact on Fertility, Menopause and Sexual Health among Pre-menopausal Women with Cancer. Journal of Cancer. 2012;3:217-225.

50. Hershberger PE, Finnegan L, Pierce PF, Scoccia B. The decision-making process of young adult women with cancer who considered fertility cryopreservation. Journal of Obstetric, Gynecologic, \& Neonatal Nursing: Clinical Scholarship for the Care of Women, Childbearing Families, \& Newborns. Jan-Feb 2013;42(1):59-69.

51. Miedema B, Easley J, Robinson LM. Do current cancer follow-up care practices meet the needs of young adult cancer survivors in Canada? A qualitative inquiry. Current Oncology. 2013;20(1):14-22.

52. Rabah DM, El-Nimr N, Rafe BA, Arafa MA. Fertility cryopreservation for female cancer patients: Attitudes and clinical practices of oncologists in Riyadh, Saudi Arabia. J Reprod Med. October 2012;57(5):431-434.

53. Louwe LA, ter Kuile MM, Hilders CG, et al. Oncologists' practice and attitudes regarding fertility preservation in female cancer patients: a pilot study in the Netherlands. J Psychosom Obstet Gynaecol. Sep 2013;34(3):129-132.

54. Quinn GP, Vadaparampil ST, Gwede CK, et al. Discussion of fertility preservation with newly diagnosed patients: oncologists' views. J. June 2007;1(2):146-155.

55. King JW, Davies MC, Roche N, Abraham JM, Jones AL. Fertility Preservation in Women Undergoing Treatment for Breast Cancer in the UK: A Questionnaire Study. Oncologist. Jul 2012;17(7):910-916.

56. Fuchs A, Kashanian JA, Clayman ML, et al. Pediatric Oncology Providers' Attitudes and Practice Patterns Regarding Fertility Preservation in Adolescent Male Cancer Patients. J. Pediatr. Hematol. Oncol. Mar 2016;38(2):118-122.

57. Goodwin T, Oosterhuis BE, Kiernan M, Hudson MA, Dahl GV. Attitudes and practices of pediatric oncology providers regarding fertility issues. Pediatr. Blood Cancer. Jan 2007;48(1):80-85.

58. Benedict C, Shuk E, Ford JS. Fertility Issues in Adolescent and Young Adult Oncology. Journal of Adolescent and Young Adult Oncology. 2016;5(1):48-57.

59. Dryden A, Ussher JM, Perz J. Young women's construction of their post-cancer fertility. Psychology \& Health. 2014/11/02 2014;29(11):1341-1360. 
PATIENT ONCOFERTILITY SUPPORT NEEDS

Table 1: Search Terms Utilised across All Electronic Databases

\begin{tabular}{|c|c|c|c|c|c|}
\hline Cancer & & Fertility & Health Service & & Patient-Clinician Interactions \\
\hline $\begin{array}{l}\text { Neoplasm*/ or oncology / or } \\
\text { cancer survivor/ or cancer } \\
\text { survival/ or childhood cancer }\end{array}$ & AND & $\begin{array}{l}\text { Fertility/ or infertility, female/ } \\
\text { or infertility, male/ or } \\
\text { reproduction/or reproductive AND } \\
\text { health/ or reproductive } \\
\text { physiological phenomena/ or } \\
\text { reproductive techniques/or } \\
\text { fertility preservation/or } \\
\text { sexual reproduction }\end{array}$ & $\begin{array}{l}\text { Health service*/or Health } \\
\text { knowledge, attitudes, practice/ or } \\
\text { health care utilization/ or attitude } \\
\text { to health/ or health services } \\
\text { research/ or health service needs/ } \\
\text { or health care delivery }\end{array}$ & AND & $\begin{array}{l}\text { Professional-patient relations/ or doctor } \\
\text { patient relation/ or patient satisfaction/ or } \\
\text { client satisfaction/ or patient care } \\
\text { management/ or quality health care/ or } \\
\text { health care quality, access and evaluation }\end{array}$ \\
\hline
\end{tabular}

This article is protected by copyright. All rights reserved. 
Figure 1: Flowchart of inclusion/exclusion process

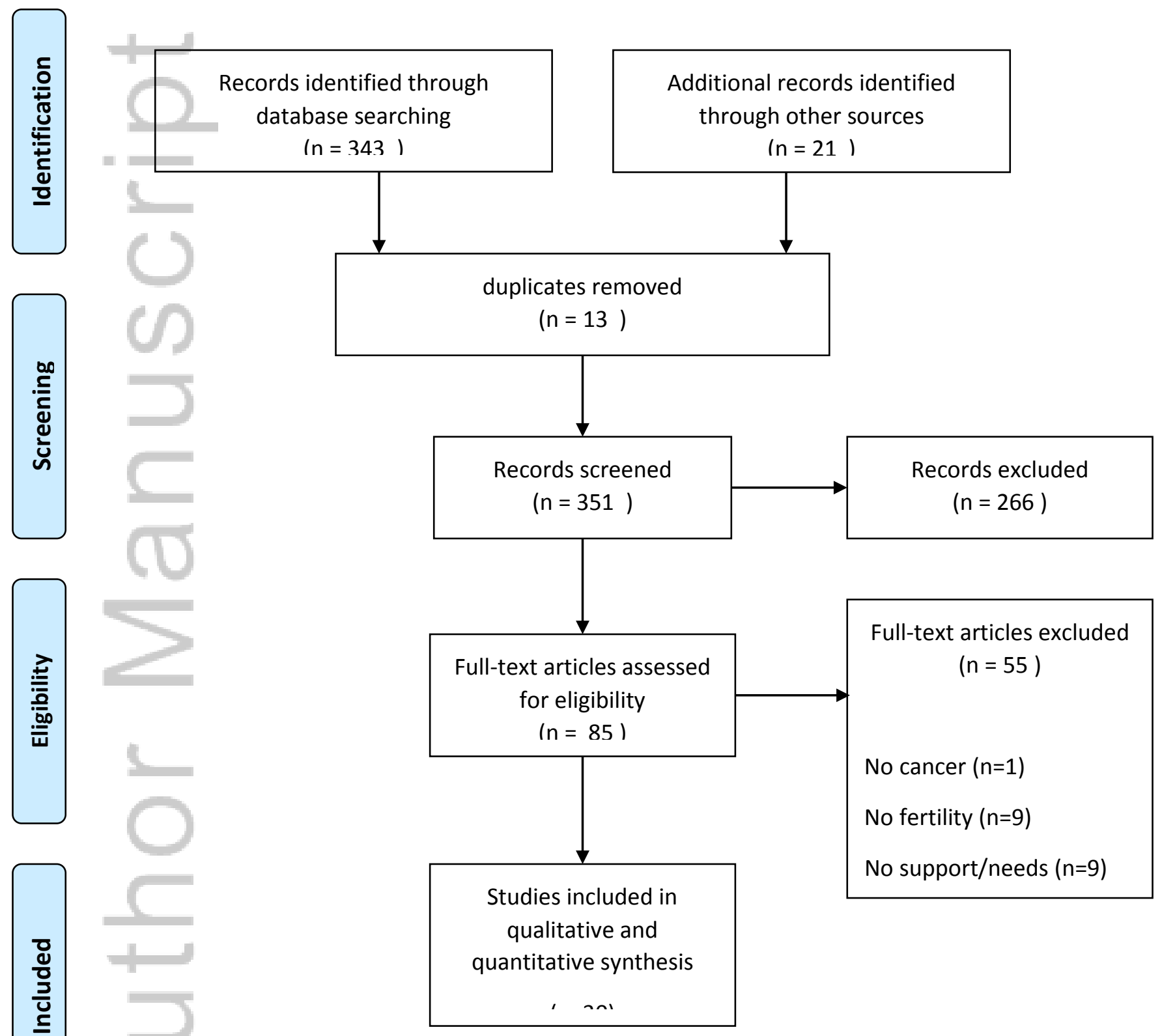

This article is protected by copyright. All rights reserved. 


\section{University Library}

\section{- M M N E R VA A gateway to Melbourne's research publications}

Minerva Access is the Institutional Repository of The University of Melbourne

Author/s:

Logan, S;Perz, J;Ussher, JM;Peate, M;Anazodo, A

Title:

A systematic review of patient oncofertility support needs in reproductive cancer patients aged 14 to $45 y e a r s$ of age

Date:

2018-02-01

Citation:

Logan, S., Perz, J., Ussher, J. M., Peate, M. \& Anazodo, A. (2018). A systematic review of patient oncofertility support needs in reproductive cancer patients aged 14 to $45 y$ years of age. PSYCHO-ONCOLOGY, 27 (2), pp.401-409. https://doi.org/10.1002/pon.4502.

Persistent Link:

http://hdl.handle.net/11343/293450 